https://doi.org/10.15407/ujpe66.8.674

D. AYEHU

Department of Physics, Wollo University

(P.O.Box 1145, Dessie, Ethiopia; e-mail: dslgnh0@gmail.com)

\title{
TWO-MODE SQUEEZED \\ AND ENTANGLED LIGHT PRODUCTION IN PARAMETRIC OSCILLATIONS
}

\begin{abstract}
We investigate the statistical and quadrature squeezings, as well as the entanglement properties, of a two-mode light generated by non-degenerate parametric oscillations coupled to a two-mode squeezed vacuum reservoir, by employing the solutions of the quantum Langevin equations. It is found that the two-mode light shows the two-mode squeezing and entanglement for all values of the time. Moreover, it is observed that the squeezed vacuum reservoir and the growing amplitude of the pump mode enhance the degrees of two-mode squeezing and entanglement. We have also shown that the amounts of squeezing and entanglement are significant in a region, where the mean photon number is higher, and the photon number correlation is lower.

Ke y w ords: two-mode light, two-mode squeezing, entanglement, photon number correlation.
\end{abstract}

\section{Introduction}

Considerable attention has been paid to squeezed states of light for the last few years [1-13]. Squeezed states of light are characterized by the reduction of quantum noise in one quadrature component of light below the vacuum or a coherent state with increased noise in the conjugate quadrature with the product of the uncertainties without violating the uncertainty relation. Having a low noise in one quadrature component, squeezed light has a potential application in the optical communication $[14,15]$, gravitation wave detection [16,17], and spectroscopic measurements [18].

One of the well-known sources of a squeezed state of light is the non-degenerate parametric oscillation, due to the inherent two-photon nature of the interaction [7]. The basic process that takes place in a non-degenerate parametric oscillation is the downconversion of a pump photon of frequency $\omega_{a}$ into a pair of photons of smaller frequencies $\omega_{b}$ and $\omega_{c}$ by the non-linear crystal. To conserve energy, we require the frequencies of the pump and down-converted photons obey the relation $\omega_{a}=\omega_{b}+\omega_{c}$. If the downconverted photons have the same frequencies, the oscillation is referred to as degenerate; otherwise, it is called non-degenerate, and the down-converted photons with different frequencies are called the signal and idler photons.

(C) D. AYEHU, 2021
It has been shown by many authors that the maximum quadrature squeezing of the cavity light generated by a parametric oscillation coupled to a vacuum reservoir following different methods is $50 \%$ below the shot noise limit [2-5]. However, the degree of squeezing more than $60 \%$ below the standard quantum limit has been achieved experimentally in a degenerate parametric oscillation operating below the threshold [19].

In recent years, the entanglement, a purely quantum measure of correlation, has attracted a great deal of interest in connection with its potential to be an essential resource for the quantum computation and communication [20], quantum dense coding [21], quantum teleportation [22], and quantum cryptography [23]. A model for the generation of a continuousvariable entanglement was developed for non-degenerate parametric oscillations. For instance, Zhang et al. [24] demonstrated experimentally that entangled light is generated in a non-degenerate parametric oscillation. Recently, two-mode squeezed and entangled light has also been realized in a non-degenerate threelevel lasers $[12,25,26]$.

In this work, we will demonstrate the statistical and quantum properties of the two-mode cavity light produced by a non-degenerate parametric oscillation coupled to a two-mode squeezed vacuum reservoir as shown in Fig. 1. In particular, the effects of the squeezed vacuum reservoir and the amplitude of the

ISSN 2071-0194. Ukr. J. Phys. 2021. Vol. 66, No. 8 
pump mode on the mean photon number sum and difference, the two-mode squeezing, and the photon number correlation of the cavity radiation are studied with the use of solutions of the quantum Langevin equations. In addition, with the aid of the same solutions, the entanglement of the cavity modes is determined using the inseparability criteria for the continuous variable state introduced in $[27,28]$.

\section{Hamiltonian and Quantum Langevin Equations}

In a non-degenerate parametric oscillation, a pump photon with a frequency $\left(\omega_{a}\right)$ splits into two highly correlated photons in a non-linear crystal called the signal and idler modes with a frequencies $\omega_{b}$ and $\omega_{c}$, respectively. The crystal is called non-linear, if it has a quadratic response to the applied electric field (pump mode).

By representing the annihilation operator for the external coherent radiation by a real constant $\beta$, the interaction of the external coherent radiation with the non-linear crystal responsible for the parametric interaction is described in the rotating wave and dipole approximations by the Hamiltonian

$H_{s}=i \lambda\left(\hat{a} \hat{b}-\hat{a}^{\dagger} \hat{b}^{\dagger}\right)$,

where $\lambda=g \beta$ is proportional to the amplitude of the pump mode, $\hat{a}$ is the annihilation operator for the signal mode, $\hat{b}$ is the annihilation operator for the idler mode, and $g$ is the measure of the coupling of a nonlinear crystal with the cavity modes. The master equation for the signal and idler modes coupled to the two-mode squeezed vacuum reservoir following the procedure described in [29] is found to be

$$
\begin{aligned}
& \frac{d \hat{\rho}}{d t}=\lambda\left(\hat{a} \hat{b} \hat{\rho}-\hat{a}^{\dagger} \hat{b}^{\dagger} \hat{\rho}-\hat{\rho} \hat{a} \hat{b}+\hat{\rho} \hat{a}^{\dagger} \hat{b}^{\dagger}\right)+ \\
& +\frac{\kappa}{2}(N+1)\left(2 \hat{a} \hat{\rho} \hat{a}^{\dagger}-\hat{a}^{\dagger} \hat{a} \hat{\rho}-\hat{\rho} \hat{a}^{\dagger} \hat{a}\right)+ \\
& +\frac{\kappa}{2} N\left(2 \hat{a}^{\dagger} \hat{\rho} \hat{a}-\hat{a} \hat{a}^{\dagger} \hat{\rho}-\hat{\rho} \hat{a} \hat{a}^{\dagger}\right)+ \\
& +\frac{\kappa}{2}(N+1)\left(2 \hat{b} \hat{\rho} \hat{b}^{\dagger}-\hat{b}^{\dagger} \hat{b} \hat{\rho}-\hat{\rho} \hat{b}^{\dagger} \hat{b}\right)+ \\
& +\frac{\kappa}{2} N\left(2 \hat{b}^{\dagger} \hat{\rho} \hat{b}-\hat{b} \hat{b}^{\dagger} \hat{\rho}-\hat{\rho} \hat{b} b^{\dagger}\right)+ \\
& +\kappa M\left(\hat{a}^{\dagger} \hat{\rho} \hat{b}^{\dagger}+\hat{b}^{\dagger} \hat{\rho} \hat{a}^{\dagger}+\hat{a} \hat{\rho} \hat{b}+\right. \\
& \left.+\hat{b} \hat{\rho} \hat{a}-\hat{a}^{\dagger} \hat{b}^{\dagger} \hat{\rho}-\hat{a} \hat{b} \hat{\rho}-\hat{\rho} \hat{a}^{\dagger} \hat{b}^{\dagger}-\hat{\rho} \hat{a} \hat{b}\right),
\end{aligned}
$$

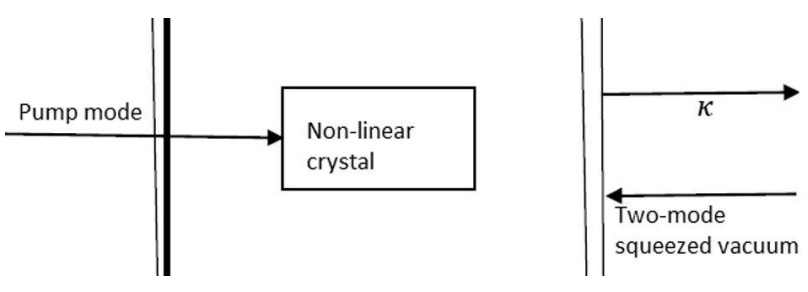

Fig. 1. Scheme of a non-degenerate parametric oscillation with $\kappa$ assumed to be the same for the signal and idler modes

where $\kappa$ is the cavity decay rate, and $N$ and $M$ are parameters that describe the effect of the squeezed vacuum reservoir and are given by

$M=\sinh (r) \cosh (r)$,

$N=\sinh ^{2}(r)$,

where $r$ is a real positive squeeze parameter.

We proceed to determine the correlation properties of the noise operators associated with the two-mode squeezed vacuum reservoir. To this end, on the basis of Eqs. (A6) and (A7), we can write

$\begin{aligned} \frac{d}{d t} \hat{a}(t) & =-\frac{\kappa}{2} \hat{a}(t)-\lambda \hat{b}^{\dagger}(t)+\hat{F}_{a}(t), \\ \frac{d}{d t} \hat{b}(t) & =-\frac{\kappa}{2} \hat{b}(t)-\lambda \hat{a}^{\dagger}(t)+\hat{F}_{b}(t),\end{aligned}$

where $\hat{F}_{a}(t)$ and $\hat{F}_{b}(t)$ are the noise operators associated with the two-mode squeezed vacuum reservoir whose correlation properties remained to be determined. We note that Eq. (A6) and the expectation value of Eq. (5) as well as Eq. (A7) and the expectation value of Eq. (6) are equal to one another, if and only if

$\left\langle\hat{F}_{a}(t)\right\rangle=\left\langle\hat{F}_{b}(t)\right\rangle=0$.

In addition, using Eqs. (5) and (6) along with the relation

$\frac{d}{d t}\langle\hat{a}(t) \hat{b}(t)\rangle=\left\langle\frac{d \hat{a}(t)}{d t} \hat{b}(t)\right\rangle+\left\langle\hat{a}(t) \frac{d \hat{b}(t)}{d t}\right\rangle$,

we have

$\frac{d}{d t}\langle\hat{a}(t) \hat{b}(t)\rangle=-\kappa\langle\hat{a}(t) \hat{b}(t)\rangle-\lambda\left\langle\hat{a}^{\dagger}(t) \hat{a}(t)\right\rangle-\lambda-$
$-\lambda\left\langle\hat{b}^{\dagger}(t) \hat{b}(t)\right\rangle+\left\langle\hat{b}(t) \hat{F}_{a}(t)\right\rangle+\left\langle\hat{a}(t) \hat{F}_{b}(t)\right\rangle$,

so that comparison of Eqs. (A10) and (9) leads to

$\left\langle\hat{b}(t) \hat{F}_{a}(t)\right\rangle+\left\langle\hat{a}(t) \hat{F}_{b}(t)\right\rangle=-\kappa M$. 
Now, multiplying the solutions of Eq. (5) from the right by $\hat{F}_{b}(t)$ and the solution of Eq. (6) from the right by $\hat{F}_{a}(t)$, considering the expectation value of the resulting expressions together with the fact that the noise operators at a certain time do not affect the cavity mode operators at the earlier time, and taking Eq. (10) into account, we see that

$\left\langle\hat{a}(t) \hat{F}_{b}(t)\right\rangle+\left\langle\hat{b}(t) \hat{F}_{a}(t)\right\rangle=\int_{0}^{t} e^{-\kappa\left(t-t^{\prime}\right) / 2} \times$

$\times\left\langle\hat{F}_{a}\left(t^{\prime}\right) \hat{F}_{b}(t)\right\rangle d t^{\prime}+\int_{0}^{t} e^{-\kappa\left(t-t^{\prime}\right) / 2}\left\langle\hat{F}_{b}\left(t^{\prime}\right) \hat{F}_{a}(t)\right\rangle d t^{\prime}$

Thus, in view of this expression and the assumption that

$\left\langle\hat{F}_{a}\left(t^{\prime}\right) \hat{F}_{b}(t)\right\rangle=\left\langle\hat{F}_{b}\left(t^{\prime}\right) \hat{F}_{a}(t)\right\rangle$,

we can readily obtain

$\left\langle\hat{F}_{a}\left(t^{\prime}\right) \hat{F}_{b}(t)\right\rangle=\left\langle\hat{F}_{b}\left(t^{\prime}\right) \hat{F}_{a}(t)\right\rangle=-\kappa M \delta\left(t-t^{\prime}\right)$.

Following a similar procedure, the correlation properties of the noise operators are found to be

$\left\langle\hat{F}_{a}^{\dagger}\left(t^{\prime}\right) \hat{F}_{a}(t)\right\rangle=\left\langle\hat{F}_{b}^{\dagger}\left(t^{\prime}\right) \hat{F}_{b}(t)\right\rangle=\kappa N \delta\left(t-t^{\prime}\right)$,

$\left\langle\hat{F}_{a}\left(t^{\prime}\right) \hat{F}_{a}^{\dagger}(t)\right\rangle=\left\langle\hat{F}_{b}\left(t^{\prime}\right) \hat{F}_{b}^{\dagger}(t)\right\rangle=\kappa(N+1) \delta\left(t-t^{\prime}\right)$,

$\left\langle\hat{F}_{a}\left(t^{\prime}\right) \hat{F}_{a}(t)\right\rangle=\left\langle\hat{F}_{b}\left(t^{\prime}\right) \hat{F}_{b}(t)\right\rangle=\left\langle F_{a}^{\dagger}\left(t^{\prime}\right) \hat{F}_{b}(t)\right\rangle=$

$=\left\langle\hat{F}_{a}\left(t^{\prime}\right) \hat{F}_{b}^{\dagger}(t)\right\rangle=0$.

We next proceed to determine the solutions of the quantum Langevin equations. To this end, making use of Eqs. (5) and (6), we can write

$\frac{d}{d t} \hat{x}_{ \pm}(t)=-1 / 2 \eta_{ \pm} \hat{x}_{ \pm}+\hat{F}_{ \pm}(t)$

where

$\hat{x}_{ \pm}(t)=\hat{a}(t) \pm \hat{b}^{\dagger}(t)$

$\eta_{ \pm}=\kappa \pm 2 \lambda$

$\hat{F}_{ \pm}(t)=\hat{F}_{a}(t) \pm \hat{F}_{b}^{\dagger}(t)$.

On the basis of Eqs. (17) and (19), the equation of evolution for $x_{-}(t)$ has no solution for $\kappa<$
$2 \lambda$. We then identify $\kappa=2 \lambda$ as the threshold condition. Thus, for $2 \lambda<\kappa$, the solution of Eq. (17) is given by

$\hat{x}_{ \pm}(t)=\hat{x}_{ \pm}(0) e^{-\eta_{ \pm} t / 2}+\int_{0}^{t} e^{-\eta_{ \pm}\left(t-t^{\prime}\right) / 2} \hat{F}_{ \pm}\left(t^{\prime}\right) d t^{\prime}$.

Now, on account of this expression and Eq. (18), we easily find

$\hat{a}(t)=A_{+}(t) \hat{a}(0)+A_{-}(t) \hat{b}^{\dagger}(0)+R_{+}(t)+R_{-}(t)$,

$\hat{b}(t)=A_{-}(t) \hat{a}^{\dagger}(0)+A_{+}(t) \hat{b}(0)+R_{+}^{\dagger}(t)-R_{-}^{\dagger}(t)$,

where

$A_{ \pm}=\frac{1}{2}\left[e^{-\eta_{+} t / 2} \pm e^{-\eta_{-} t / 2}\right]$

$\hat{R}_{ \pm}(t)=\frac{1}{2}\left[\int_{0}^{t} e^{-\eta_{ \pm}\left(t-t^{\prime}\right) / 2}\left(\hat{F}_{a}\left(t^{\prime}\right) \pm \hat{F}_{b}^{\dagger}\left(t^{\prime}\right)\right] d t^{\prime}\right.$.

We now calculate the various expectation values of the cavity mode operators. In view of Eq. (22) and its adjoint, one can write

$\left\langle\hat{a}^{\dagger} \hat{a}\right\rangle=A_{+}^{2}\left\langle\hat{a}^{\dagger}(0) \hat{a}(0)\right\rangle+A_{+} A_{-}(t)\left\langle\hat{a}^{\dagger}(0) \hat{b}^{\dagger}(0)\right\rangle+$

$+A_{+}\left\langle\hat{a}^{\dagger}(0) R_{+}(t)\right\rangle+A_{+}\left\langle\hat{a}^{\dagger}(0) R_{-}(t)\right\rangle+$

$+A_{-} A_{+}\langle\hat{b}(0) \hat{a}(0)\rangle+A_{-}^{2}\left\langle\hat{b}(0) \hat{b}^{\dagger}(0)\right\rangle+$

$+A_{-}(t)\left\langle\hat{b}(0) R_{+}(t)\right\rangle+A_{-}(t)\left\langle\hat{b}(0) R_{-}(t)\right\rangle+$

$+A_{+}\left\langle R_{+}^{\dagger} \hat{a}(0)\right\rangle+A_{-}(t)\left\langle R_{+}^{\dagger}(t) \hat{b}^{\dagger}(0)\right\rangle+$

$+\left\langle R_{+}^{\dagger}(t) R_{+}(t)\right\rangle+\left\langle R_{+}^{\dagger}(t) R_{-}(t)\right\rangle+$

$+A_{+}(t)\left\langle R_{-}^{\dagger}(t) \hat{a}(0)\right\rangle+A_{-}(t)\left\langle R_{-}^{\dagger}(t) \hat{b}^{\dagger}(0)\right\rangle+$

$+\left\langle R_{-}^{\dagger}(t) R_{+}(t)\right\rangle+A_{+}(t)\left\langle R_{-}^{\dagger}(t) \hat{a}(0)\right\rangle+\left\langle R_{-}^{\dagger}(t) R_{-}(t)\right\rangle$.

Based on the fact that the noise operator at a certain time does not affect the cavity mode operator at an earlier time and on the assumption that the cavity light is initially in a two-mode vacuum state along with the correlation properties described by Eqs. (13)-(16), we get

$\left\langle\hat{a}^{\dagger} \hat{a}\right\rangle=\frac{\kappa(2 N+1-2 M)\left(1-e^{\left.-\eta_{+} t\right)}\right.}{4 \eta_{+}}+$

$+\frac{\kappa(2 N+1+2 M)\left(1-e^{\left.-\eta_{-} t\right)}\right.}{4 \eta_{-}}+$

$+\frac{1}{4}\left(e^{-\eta_{+} t}+e^{-\eta_{-} t}-2\right)$.

ISSN 2071-0194. Ukr. J. Phys. 2021. Vol. 66, No. 8 
It can also be readily established in a similar way that $\left\langle\hat{b}^{\dagger} \hat{b}\right\rangle=\frac{\kappa(2 N+1-2 M)\left(1-\exp -\eta_{+} t\right)}{4 \eta_{+}}+$ $+\frac{\kappa(2 N+1+2 M)\left(1-\exp -\eta_{-} t\right)}{4 \eta_{-}}+$

$+\frac{1}{4}\left(e^{-\eta_{+} t}+e^{-\eta_{-} t}-2\right)$,

$\langle\hat{a}(t) \hat{b}(t)\rangle=\frac{\kappa(2 N+1-2 M)\left(1-e^{-\eta_{+} t}\right)}{4 \eta_{+}}=$

$=-\frac{\kappa(2 N+1+2 M)\left(1-e^{-\eta_{-} t}\right)}{4 \eta_{-}}+$

$+\frac{1}{4}\left(e^{-\eta_{+} t}-e^{-\eta-t}\right)$,

$\left\langle\hat{a}(t) \hat{b}^{\dagger}(t)\right\rangle=\left\langle\hat{a}^{\dagger}(t) \hat{b}(t)\right\rangle=0$,

$\left\langle\hat{a}^{2}(t)\right\rangle=\left\langle\hat{b}^{2}(t)\right\rangle=0$.

\section{Two-Mode Quadrature Squeezing}

We next proceed to determine the quadrature variance and the quadrature squeezing of the two-mode cavity light produced by a non-degenerate parametric oscillation coupled to the two-mode squeezed vacuum reservoir. We represent the two-mode light by the operator $\hat{c}$ defined by

$\hat{c}(t)=\hat{a}(t)+\hat{b}(t)$.

The squeezing properties of a two-mode light are described by the plus and minus quadrature operators defined as

$\hat{c}_{+}=\hat{c}(t)+\hat{c}^{\dagger}(t)$

$\hat{c}_{-}=i\left(\hat{c}^{\dagger}(t)-\hat{c}(t)\right)$.

One can readily verify that

$\left[\hat{c}_{+}, \hat{c}_{-}\right]=4 i$.

It then follows that a two-mode light is said to be in a two-mode squeezed state, if either $\Delta \hat{c}_{+}>\sqrt{2}$ and $\Delta \hat{c}_{-}<\sqrt{2}$ or $\Delta \hat{c}_{+}<\sqrt{2}$ and $\Delta \hat{c}_{-}>\sqrt{2}$ such that $\Delta \hat{c}_{+} \Delta \hat{c}_{-} \geq 2$. In view of Eqs. (5), (6), and (32), we can easily verify that

$\frac{d}{d t} \hat{c}_{+}(t)=-\frac{\eta_{+}}{2} \hat{c}_{+}(t)+\hat{F}_{+}(t)$,

$\frac{d}{d t} \hat{c}_{-}(t)=-\frac{\eta_{-}}{2} \hat{c}_{-}(t)+i \hat{F}_{-}(t)$, where

$\hat{F}_{ \pm}=\hat{F}_{a}^{\dagger}(t) \pm \hat{F}_{a}(t)+\hat{F}_{b}^{\dagger}(t) \pm \hat{F}_{b}(t)$.

Thus, the solutions of Eqs. (36) and (37) can be written as

$\hat{c}_{+}(t)=\hat{c}_{+}(0) e^{-\eta_{+} t / 2}+\int_{0}^{t} e^{-\eta_{+}\left(t-t^{\prime}\right) / 2} \times$

$\times\left(\hat{F}_{a}^{\dagger}\left(t^{\prime}\right)+\hat{F}_{a}\left(t^{\prime}\right)+\hat{F}_{b}^{\dagger}\left(t^{\prime}\right)+\hat{F}_{b}\left(t^{\prime}\right)\right) d t^{\prime}$,

$\hat{c}_{-}(t)=\hat{c}_{-}(0) e^{-\eta_{-} t / 2}+i \int_{0}^{t}\left(e^{-\eta_{-}\left(t-t^{\prime}\right) / 2} \times\right.$

$\times\left[\hat{F}_{a}^{\dagger}\left(t^{\prime}\right)-\hat{F}_{a}\left(t^{\prime}\right)+\hat{F}_{b}^{\dagger}\left(t^{\prime}\right)-\hat{F}_{b}\left(t^{\prime}\right)\right] d t^{\prime}$.

The variances of the quadrature operators are defined by

$\left(\Delta \hat{c}_{ \pm}(t)\right)^{2}=\left\langle\hat{c}_{ \pm}^{2}(t)\right\rangle-\left\langle\hat{c}_{ \pm}(t)\right\rangle^{2}$.

Now, considering the expectation values of Eqs. (39) and (40) and the assumption that the cavity light is initially in a two-mode vacuum state along with the correlation properties described in Eq. (7), we have

$\left\langle\hat{c}_{+}(t)\right\rangle=0$

$\left\langle\hat{c}_{-}(t)\right\rangle=0$.

Moreover, using Eqs. (39) and (40) together with Eqs. (13)-(16), we arrive at

$$
\begin{aligned}
& \left\langle\hat{c}_{+}^{2}(t)\right\rangle=2 e^{-\eta_{+} t / 2}+ \\
& +\frac{\kappa(4 N+2-4 M)\left(1-e^{-\eta_{+} t}\right)}{\eta_{+}}, \\
& \left\langle\hat{c}_{-}^{2}(t)\right\rangle=2 e^{-\eta_{-} t / 2}+ \\
& +\frac{\kappa(4 N+2+4 M)\left(1-e^{-\eta_{-} t}\right)}{\eta_{-}} .
\end{aligned}
$$

Now, by employing Eqs. (42), (43), (44) and (45), we reduce the variances of the quadrature operators to

$$
\begin{aligned}
& \left(\Delta \hat{c}_{+}(t)\right)^{2}=2 e^{-\eta_{+} t / 2}+ \\
& +\frac{\kappa(4 N+2-4 M)\left(1-e^{-\eta_{+} t}\right)}{\eta_{+}} \\
& \left(\Delta \hat{c}_{-}(t)\right)^{2}=2 e^{-\eta_{-} t / 2}+ \\
& +\frac{\kappa(4 N+2+4 M)\left(1-e^{-\eta_{-} t}\right)}{\eta_{-}} .
\end{aligned}
$$




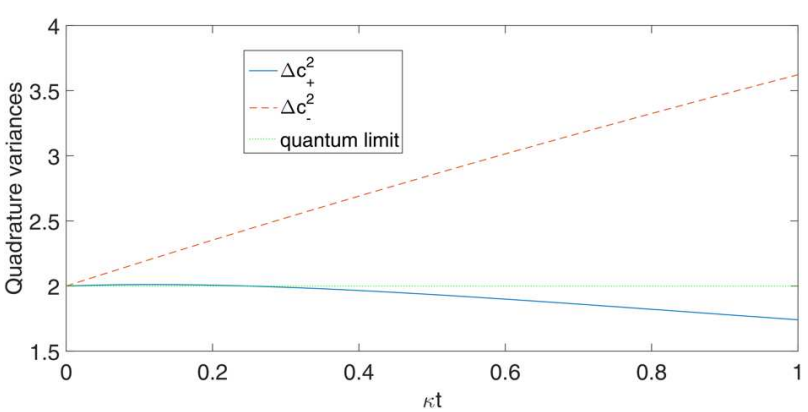

Fig. 2. Plots of the variances of the plus quadrature [Eq. (48)] (dashed curve) and minus quadrature [Eq. (49)] (solid curve) operators versus $\kappa t$ for $\frac{\lambda}{\kappa}=0.4$ and $r=0$

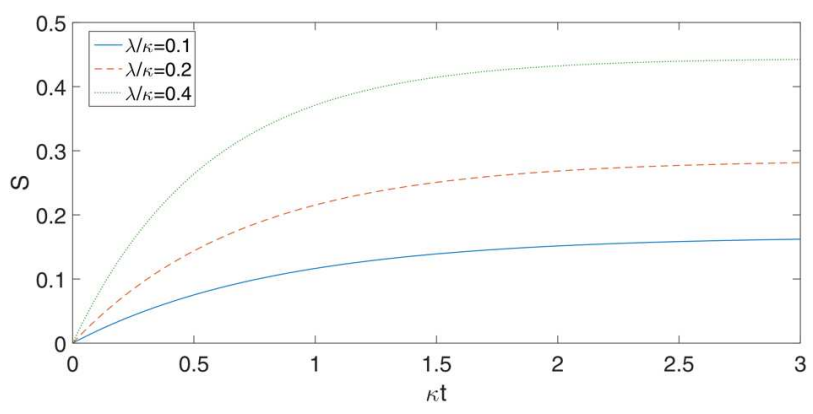

Fig. 3. Plots of the quadrature squeezing [Eq. (54)] versus $\kappa t$ for $\mathrm{r}=0$ and different values of $\lambda / \kappa$

Making use of Eqs. (3), (4), and (19), we find

$$
\begin{aligned}
& \left(\Delta \hat{c}_{+}(t)\right)^{2}=2 e^{-(\kappa+2 \lambda) t / 2}+ \\
& +\frac{2 \kappa e^{-2 r}}{\kappa+2 \lambda}\left(1-e^{-(\kappa+2 \lambda) t}\right), \\
& \left(\Delta \hat{c}_{-}(t)\right)^{2}=2 e^{-(\kappa-2 \lambda) t / 2}+ \\
& +\frac{2 \kappa e^{2 r}}{\kappa-2 \lambda}\left(1-e^{-(\kappa-2 \lambda) t}\right) .
\end{aligned}
$$

In Fig. 2, we plot the variances of the plus and minus quadrature operators versus the time. The horizontal dotted line in this figure represents the coherent or vacuum state level. From Fig. 2, we see that the variance of the plus quadrature operator is below the shot noise limit, while the variance of the minus quadrature operator is above the shot noise limit. In general, one can see that the cavity light beams have unequal fluctuations. In the minus quadrature, the fluctuation is increased or stretched, and, in the plus quadrature, the fluctuation is reduced or squeezed. We then note that the squeezing occurs in the plus quadrature.

\section{8}

It is fascinating to consider some special cases for the system under consideration. For example, in the absence of the two-mode squeezed vacuum reservoir, the system reduces to a non-degenerate parametric oscillation coupled to a two-mode vacuum reservoir. Hence, upon putting $N=M=0$ in Eqs. (46) and (47), the quadrature variances reduce to

$$
\begin{aligned}
& \left(\Delta c_{+}\right)^{2}=2 e^{-(\kappa+2 \lambda) t}+\frac{2 \kappa}{\kappa+2 \lambda}\left(1-e^{-(\kappa+2 \lambda) t}\right), \\
& \left(\Delta c_{-}\right)^{2}=2 e^{-(\kappa-2 \lambda) t}+\frac{2 \kappa}{\kappa-2 \lambda}\left(1-e^{-(\kappa-2 \lambda) t}\right) .
\end{aligned}
$$

These results are in agreement with the previous studies $[2,5,14-16]$ obtained within various approaches. In the case where the parametric oscillation, $\lambda$, and the two-mode squeezed vacuum reservoir, $r$, are absent, the system is in a cavity mode in a two-mode vacuum state, and the quadrature variances of this system take the form

$\left(\Delta c_{+}\right)^{2}=\left(\Delta c_{-}\right)^{2}=2$.

The quadrature squeezing of the cavity light can be determined relative to the quadrature variance of the two-mode vacuum state by the relation $[3,13]$

$S=\frac{\left(\Delta \hat{c}_{+}\right)_{\mathrm{vac}}^{2}-\left(\Delta \hat{c}_{+}\right)^{2}}{\left(\Delta \hat{c}_{+}\right)_{\mathrm{vac}}^{2}}$

where $\left(\Delta \hat{c}_{+}\right)_{\mathrm{vac}}^{2}$ is the quadrature variance of the twomode vacuum state. Thus, by substituting Eqs. (48) and (52) into Eq. (53), we obtain

$$
\begin{aligned}
& S=1-e^{-\kappa t(1+2 \lambda / \kappa)}-\frac{e^{-2 r}}{(1+2 \lambda / \kappa)} \times \\
& \times\left(1-e^{-\kappa t(1+2 \lambda / \kappa)}\right) .
\end{aligned}
$$

As can be seen from Figs. 3 and 4, the two-mode light shows a considerable two-mode squeezing for all values of the time. It is expected that the cause for the two-mode squeezing is the correlation between the two cavity modes. This correlation arises due to the coherence of the external radiation (the pump mode) before the down-conversion processes. We begin by investigating the dependence of the two-mode squeezing on the amplitude of the pump mode. It is observed from Fig. 3 that a large value of the amplitude of the pump mode results in a high suppression of a noise below the standard quantum limit. Next, we proceed to demonstrate how the two-mode squeezed

ISSN 2071-0194. Ukr. J. Phys. 2021. Vol. 66, No. 8 
vacuum reservoir affects the degree of squeezing of the two-mode light. Figure 4 shows the plot of the two-mode squeezing versus $\kappa t$ for different values of the squeeze parameter, $r$. It is easy to see from Fig. 4 that the squeeze parameter has the effect of increasing the degree of squeezing of the two-mode cavity light. At the threshold and at the steady state, it is also observed that the maximum quadrature squeezing of the two-mode light is $72.5 \%$ below the standard quantum limit for $r=0.3$.

\section{Entanglement Properties of the Cavity Modes}

Here, we consider the continuous variable entanglement between the two cavity modes. A composite system in which its state cannot be factored as a product of states of its constituents is referred to as an entangled system. That is,

$|\psi\rangle \neq \sum_{i}\left|\psi_{i}^{(1)}\right\rangle \bigotimes\left|\psi_{i}^{(2)}\right\rangle$.

Several authors have proposed various entanglement criteria for continuous-variable states [27-31]. In this study, we employ the entanglement criteria introduced by Duan-Giedke-Cirac-Zoller (DGCZ) and Hillery-Zubairy (HZ) to investigate the entanglement properties of the two cavity modes.

\subsection{Hillery-Zubairy criterion}

In the Hillery-Zubairy (HZ) criteria, the state of a two-mode system is said to be entangled, if the condition

$E_{H Z} \equiv\left\langle\hat{n}_{1}\right\rangle\left\langle\hat{n}_{2}\right\rangle-\left|\left\langle\hat{a}_{1} \hat{a}_{2}\right\rangle\right|^{2}<0$

is satisfied. In other words, for the cavity modes to be entangled, the product of the mean photon number of the cavity modes should be smaller than the squared modulus of the intermode correlations. Now because of Eqs. (27), (28) and (29), we see that

$$
\begin{aligned}
& E_{\mathrm{HZ}} \equiv\left(\frac{\kappa e^{-2 r}\left(1-e^{-(\kappa+2 \lambda) t}\right)}{4(\kappa+2 \lambda)}+\right. \\
& +\frac{\kappa e^{2 r}\left(1-e^{-(\kappa-2 \lambda) t}\right)}{4(\kappa-2 \lambda)}+ \\
& \left.+\frac{1}{4}\left(e^{-(\kappa+2 \lambda) t}+e^{-(\kappa-2 \lambda) t}-2\right)\right)^{2}-
\end{aligned}
$$

ISSN 2071-0194. Ukr. J. Phys. 2021. Vol. 66, No. 8

$$
\begin{aligned}
& -\left(\frac{\kappa e^{-2 r}\left(1-e^{-(\kappa+2 \lambda) t}\right)}{4(\kappa+2 \lambda)}-\right. \\
& -\frac{\kappa e^{2 r}\left(1-e^{-(\kappa-2 \lambda) t}\right)}{4(\kappa-2 \lambda)}+ \\
& \left.+\frac{1}{4}\left(e^{-(\kappa+2 \lambda) t}-e^{-(\kappa-2 \lambda) t}\right)\right)^{2}<0 .
\end{aligned}
$$

Figure 5 shows how the amplitude of the pump mode influences the entanglement of the cavity modes. We note from this figure that the cavity modes are entangled at all times for the given parameters, and the degree of entanglement increases with the time. We then infer that the entanglement is robust at a steady state. Moreover, we see from Fig. 5 that the degree of entanglement increases with the amplitude of the pump mode. We then anticipate that increasing the amplitude of the pump mode leads to an increase in the correlation of the signal and idler photons. In Fig. 6, we plot $E_{\mathrm{HZ}}$ as a function of $\kappa t$ for different values of the squeeze parameter. We note from this figure that the squeeze parameter has the effect of increasing the degree of entanglement.

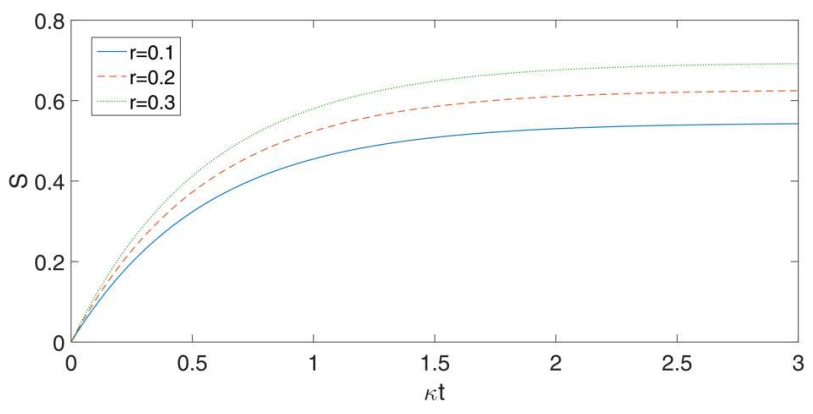

Fig. 4. Plots of the quadrature squeezing [Eq. (54)] versus $\kappa t$ for $\lambda / \kappa=0.4$ and different values of $r$

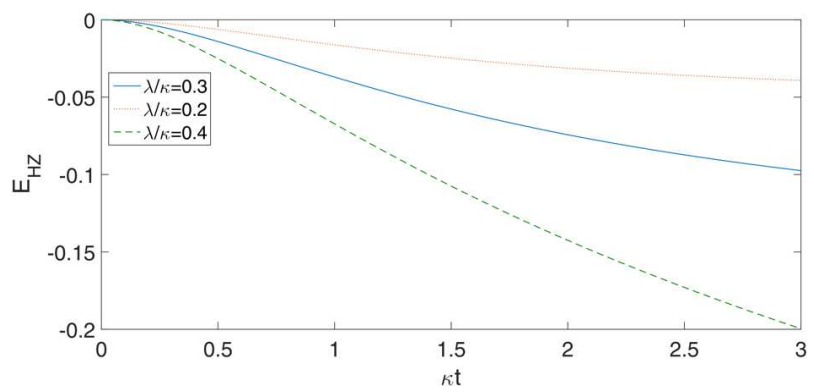

Fig. 5. Plots of Eq. (57) versus $\kappa t$ for $r=0$ and different values of $\lambda / \kappa$ 


\section{2. $D G C Z$ criterion}

According to the DGCZ criteria, the state of a twomode system is known to be entangled, if the variances of two EPR-like operators, $\hat{u}$ and $\hat{v}$, of a two mode system satisfy the inequality

$(\Delta u)^{2}+(\Delta v)^{2}<4$,

where

$\hat{u}=\hat{x}_{a}+\hat{x}_{b}$,

$\hat{v}=\hat{p}_{a}-\hat{p}_{b}$,

and

$\hat{x}_{k}=\hat{k}+\hat{k}^{\dagger}$,

$\hat{p}_{k}=\frac{\hat{k}-\hat{k}^{\dagger}}{i}$

with $(k=a, b)$ are the quadrature operators for the two cavity modes. Making use of Eqs. (59)-(62), it can be easily established that

$\Delta u^{2}=\Delta v^{2}=2\left[1+\left\langle\hat{a}^{\dagger} \hat{a}\right\rangle+\left\langle\hat{b}^{\dagger} \hat{b}\right\rangle+\langle\hat{a} \hat{b}\rangle+\left\langle\hat{a}^{\dagger} \hat{b}^{\dagger}\right\rangle\right]$

Thus, in view of Eq. (63), the sum of the fluctuations of the pair of EPR-like operators turns out to be

$\Delta u^{2}+\Delta v^{2}=4\left[1+\left\langle\hat{a}^{\dagger} \hat{a}\right\rangle+\left\langle\hat{b}^{\dagger} \hat{b}\right\rangle+\langle\hat{a} \hat{b}\rangle+\left\langle\hat{a}^{\dagger} \hat{b}^{\dagger}\right\rangle\right]$

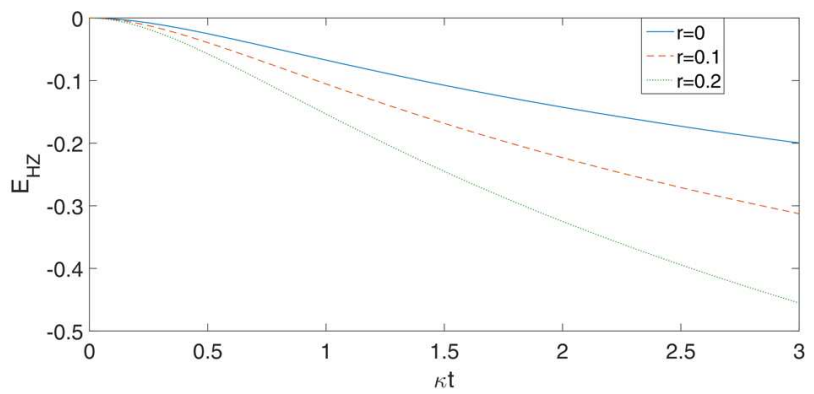

Fig. 6. Plots of Eq. (57) versus $\kappa t$ for $\lambda / \kappa=0.4$ and different values of $r$

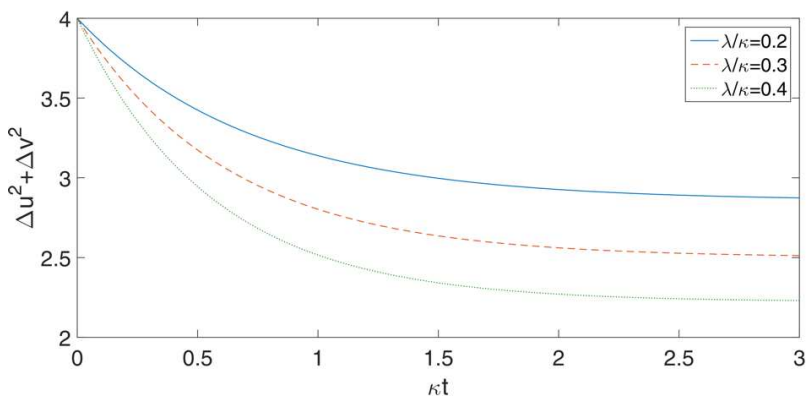

Fig. 7. Plots of $\Delta u^{2}+\Delta v^{2}$ [Eq. (66)] versus $\kappa t$ for $r=0$ and different values of $\lambda / \kappa$
Hence, in view of Eqs. (27)-(29), the fluctuations of the EPR-like operators can be put in the form

$\Delta u^{2}+\Delta v^{2}=4 e^{-\eta_{+} t}+$

$+4 \frac{\kappa(4 N+2-4 M)\left(1-e^{-\eta_{+} t}\right)}{\eta_{+}}$.

With regard for Eqs. (3), (4), and (19), we have

$\Delta u^{2}+\Delta v^{2}=4 e^{-(\kappa+2 \lambda) t}+$

$+\frac{4 \kappa e^{-2 r}}{\kappa+2 \lambda}\left(1-e^{-(\kappa+2 \lambda) t}\right)$.

Based on the relation described by Eq. (58), it is easy to see from Figs. 7 and 8 that the two-mode light produced by a non-degenerate parametric oscillation shows the entanglement for all values of the time. It is expected that the coherence of the pump mode (before the down-conversion process) incident on the nonlinear crystal is the cause for the correlation of the signal and idler photons, which leads to the entanglement of the two cavity modes. This means that even though the pump photon is destroyed in the downconversion process, it is unable to eliminate the coherence of the pump mode. It is clearly shown in Figs. 7 and 8 that the degree of entanglement significantly depends on the amplitude of the pump mode, $\lambda$, and the two-mode squeezed vacuum reservoir, $r$. We note from these two figures that the degree of entanglement increases with the time, as in the case of the HZ criteria. Moreover, the two-mode squeezed vacuum reservoir and the growth of the amplitude of the pump mode enhance the degree of entanglement between the signal and idler modes. In addition, by comparing Figs. 3 and 7, as well as Figs. 4 and 8, we see that the amounts of squeezing and entanglement vary in the same fashion. We can then infer that the degrees of squeezing and entanglement are proportional. In general, on the basis of the two aforementioned entanglement criteria, the two cavity modes exhibit the entanglement for all values of the time.

\section{Mean Photon}

\section{Number Sum and Difference}

In this section, we calculate the mean photon number sum and difference for a two-mode light. The mean photon number sum and difference of the two-mode light are expressible as

$\langle\hat{n}(t)\rangle=\left\langle\hat{a}^{\dagger}(t) \hat{a}(t)\right\rangle \pm\left\langle\hat{b}^{\dagger}(t) \hat{b}(t)\right\rangle$.

ISSN 2071-0194. Ukr. J. Phys. 2021. Vol. 66, No. 8 
Now, in view of Eqs. (27) and (28), the mean of the photon number sum for the two-mode cavity light is as follows:

$$
\begin{aligned}
& \left\langle\hat{n}_{+}(t)\right\rangle=\frac{\kappa(2 N+1-2 M)\left(1-\exp -\eta_{+} t\right)}{2 \eta_{+}}+ \\
& +\frac{\kappa(2 N+1+2 M)\left(1-\exp -\eta_{-} t\right)}{2 \eta_{-}}
\end{aligned}
$$

Making use of Eqs. (3) and (4), we find

$$
\begin{aligned}
& \left\langle\hat{n}_{+}(t)\right\rangle=\frac{\kappa e^{-2 r}}{2(\kappa+2 \lambda)}\left(1-e^{-(\kappa+2 \lambda) t}\right)+ \\
& +\frac{\kappa e^{2 r}}{2(\kappa-2 \lambda)}\left(1-e^{-(\kappa-2 \lambda) t}\right) .
\end{aligned}
$$

In Fig. 9, we plot the mean photon number sum versus $\kappa t$ for different values of the squeeze parameter, $r$. It is not difficult to see from this figure that the mean photon number sum increases with the squeeze parameter, $r$. We have also shown that the degrees of entanglement and squeezing increase with the squeeze parameter, $r$. We note that a more bright light is generated by the system under consideration in regions, where the significant degrees of squeezing and entanglement are observed. Moreover, the mean photon number sum increases with the time.

It can also be verified, by employing Eqs. (27) and (28), that the mean photon number difference for the two-mode cavity light takes the form

$\left\langle\hat{n}_{-}(t)\right\rangle=0$.

This is so, because the signal and idler photons are produced in pairs in the down-conversion process.

\section{The Photon Number Correlation}

The photon number correlation for two modes of light can be expressed as

$g\left(\hat{n}_{a}, \hat{n}_{b}\right)=\frac{\left\langle\hat{n}_{a} \hat{n}_{b}\right\rangle}{\left\langle\hat{n}_{a}\right\rangle\left\langle\hat{n}_{b}\right\rangle}$,

with

$\left\langle\hat{n}_{a} \hat{n}_{b}\right\rangle=\left\langle\hat{a}^{\dagger}(t) \hat{a}(t) \hat{b}^{\dagger}(t) \hat{b}(t)\right\rangle$.

Because $\hat{a}(t)$ and $\hat{b}(t)$ are Gaussian variables with a vanishing mean, we have

$g\left(\hat{n}_{a}, \hat{n}_{b}\right)=1+\frac{\langle\hat{a}(t) \hat{b}(t)\rangle^{2}}{\left\langle\hat{a}^{\dagger}(t) \hat{a}(t)\right\rangle\left\langle\hat{b}^{\dagger}(t) \hat{b}(t) .\right\rangle}$

ISSN 2071-0194. Ukr. J. Phys. 2021. Vol. 66, No. 8

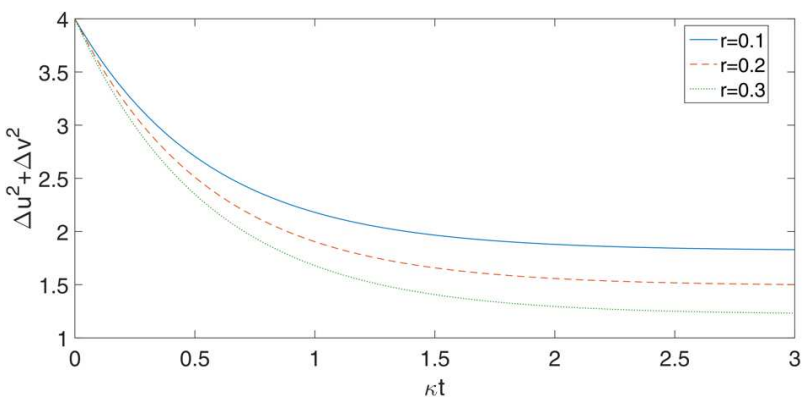

Fig. 8. Plots of $\Delta u^{2}+\Delta v^{2}$ [Eq. (66)] versus $\kappa t$ for $\lambda / \kappa=0.4$ and different values of $r$

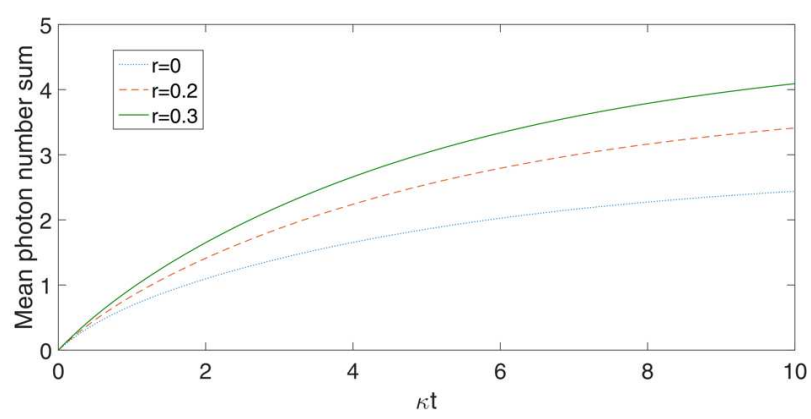

Fig. 9. Plots of the mean photon number sum [Eq. (69)] versus $\kappa t$ for $\lambda / \kappa=0.4$ and different values of $r$

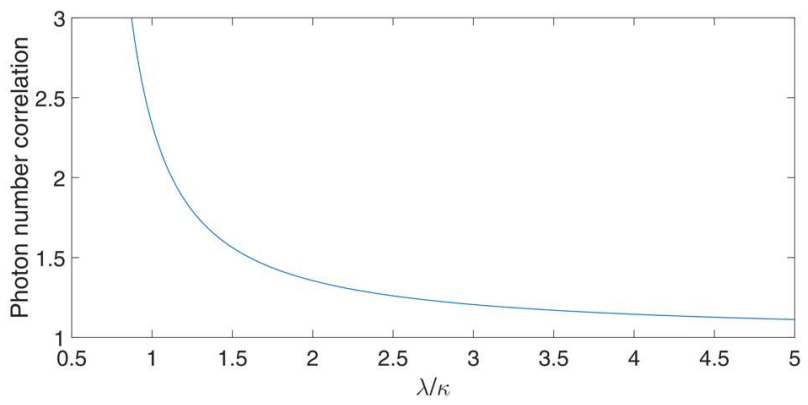

Fig. 10. Plot of the photon number correlation at steady state [Eq. (73)] versus $\lambda / \kappa$ for $r=0$

It is seen from Fig. 10 that the photon number correlation decreases with increasing the amplitude of the pump mode, $\lambda$. However, we have found that the degrees of squeezing and entanglement increase with the amplitude of the pump mode, $\lambda$. We see that the photon number correlation is minimum in regions, where the degrees of two-mode squeezing and entanglement are maximum.

\section{Conclusions}

We have considered a non-degenerate parametric oscillation coupled to a squeezed vacuum reservoir. 
Employing the master equation and applying the solutions of the quantum Langevin equations, we have studied the quadrature squeezing and the entanglement properties of the two-mode light. Moreover, using the same solutions, we determine the mean photon number sum, the mean photon number difference, and the photon number correlation of the twomode light.

It is observed that the two-mode light is in a twomode squeezed state with the maximum quadrature squeezing being $72.5 \%$ below the coherent or vacuum state level for $r=0.3$. We have also shown that the amplitude of the pump mode and the injected squeezed light have the effect of increasing the mean photon number sum and decreasing the photon number correlation of the two-mode light. It is also indicated that the photon number difference happens to be zero, since the signal and idler photons are generated in pairs in the down-conversion process of the parametric oscillation. In addition, our results show that the amounts of two-mode squeezing and entanglement are enhanced by the growth of the amplitude of the pump mode and the squeezed vacuum reservoir. We have also seen that the degrees of squeezing and entanglement are maximum in regions, where the mean photon number sum is higher, and the photon number correlation is lower. Moreover, we have noticed that the degrees of squeezing and entanglement have a direct relationship.

\section{APPENDIX.}

\section{Equation of evolution of the cavity mode operators}

In this appendix, we wish to establish the equation of evolution of the cavity mode operators using the master equation. To this end, employing the relation

$\frac{d}{d t}\langle\hat{a}\rangle=\operatorname{Tr}\left(\frac{d \hat{\rho}}{d t} \hat{a}\right)$,

along with Eq. (2), we can write

$\frac{d}{d t}\langle\hat{a}(t)\rangle=\lambda \operatorname{Tr}\left(\hat{a} \hat{b} \hat{\rho} \hat{a}-\hat{a}^{\dagger} \hat{b}^{\dagger} \hat{\rho} \hat{a}-\hat{\rho} \hat{a} \hat{b} \hat{a}+\hat{\rho} \hat{a}^{\dagger} \hat{b}^{\dagger} \hat{a}\right)+$

$+\frac{\kappa}{2}\left(N_{a}+1\right) \operatorname{Tr}\left(2 \hat{a} \hat{\rho} \hat{a}^{\dagger} \hat{a}-\hat{a}^{\dagger} \hat{a} \hat{\rho} \hat{a}-\hat{\rho} \hat{a}^{\dagger} \hat{a} \hat{a}\right)+$

$+\frac{\kappa}{2} N_{a} \operatorname{Tr}\left(2 \hat{a}^{\dagger} \hat{\rho} \hat{a} \hat{a}-\hat{a} \hat{a}^{\dagger} \hat{\rho} \hat{a}-\hat{\rho} \hat{a} \hat{a}^{\dagger} \hat{a}\right)+$

$+\frac{\kappa}{2}\left(N_{b}+1\right) \operatorname{Tr}\left(2 \hat{b} \hat{\rho} \hat{b}^{\dagger} \hat{a}-\hat{b}^{\dagger} \hat{b} \hat{\rho} \hat{a}-\hat{\rho} \hat{b}^{\dagger} \hat{b} \hat{a}\right)+$

$+\frac{\kappa}{2} N_{b} \operatorname{Tr}\left(2 \hat{b}^{\dagger} \hat{\rho} \hat{b} \hat{a}-\hat{b} \hat{b}^{\dagger} \hat{\rho} \hat{a}-\hat{\rho} \hat{b} \hat{b}^{\dagger} \hat{a}\right)+$

$+\kappa M \operatorname{Tr}\left(\hat{a}^{\dagger} \hat{\rho} \hat{b}^{\dagger} \hat{a}+\hat{b}^{\dagger} \hat{\rho} \hat{a}^{\dagger} \hat{a}+\right.$

682 $\left.+\hat{a} \hat{\rho} \hat{b} \hat{a}+\hat{b} \hat{\rho} \hat{a} \hat{a}-\hat{a}^{\dagger} \hat{b}^{\dagger} \hat{\rho} \hat{a}-\hat{a} \hat{b} \hat{\rho} \hat{a}-\hat{\rho} \hat{a}^{\dagger} \hat{b}^{\dagger} \hat{a}-\hat{\rho} \hat{a} \hat{b} \hat{a}\right)$.

Applying the cyclic property of the trace operation along with the commutation relation

$\left[\hat{a}, \hat{a}^{\dagger}\right]=\left[\hat{b}, \hat{b}^{\dagger}\right]=1$,

$[\hat{a}, \hat{b}]=\left[\hat{a}^{\dagger}, \hat{b}^{\dagger}\right]=\left[\hat{a}, \hat{b}^{\dagger}\right]=\left[\hat{a}^{\dagger}, \hat{b}\right]=0$,

we have

$\frac{d}{d t}\langle\hat{a}(t)\rangle=-\lambda \operatorname{Tr}\left(\hat{\rho} \hat{b}^{\dagger}\right)-\frac{\kappa}{2}\left(N_{a}+1\right) \operatorname{Tr}(\hat{\rho} \hat{a})+$

$+\frac{\kappa}{2} N_{a} \operatorname{Tr}(\hat{\rho} \hat{a})$.

Applying the trace operation to this equation, we see that

$\frac{d}{d t}\langle\hat{a}(t)\rangle=-\lambda\left\langle\hat{b}^{\dagger}(t)\right\rangle-\frac{\kappa}{2}\langle\hat{a}(t)\rangle$.

Following a similar fashion, we can easily show that

$\frac{d}{d t}\langle\hat{b}(t)\rangle=-\lambda\left\langle\hat{a}^{\dagger}(t)\right\rangle-\frac{\kappa}{2}\langle\hat{b}(t)\rangle$,

$\frac{d}{d t}\left\langle\hat{a}^{\dagger}(t) \hat{a}(t)\right\rangle=-\lambda\langle\hat{a}(t) \hat{b}(t)\rangle-\lambda\left\langle\hat{a}^{\dagger}(t) \hat{b}^{\dagger}(t)\right\rangle-$

$-\kappa\left\langle\hat{a}^{\dagger}(t) \hat{a}(t)\right\rangle+\kappa N$,

$\frac{d}{d t}\left\langle\hat{b}^{\dagger}(t) \hat{b}(t)\right\rangle=-\lambda\left\langle\hat{a}^{\dagger}(t) \hat{b}^{\dagger}(t)\right\rangle-\lambda\langle\hat{a}(t) \hat{b}(t)\rangle-$

$-\kappa\left\langle\hat{b}^{\dagger}(t) \hat{b}(t)+\kappa N\right.$,

$\frac{d}{d t}\langle\hat{a}(t) \hat{b}(t)\rangle=-\lambda\left\langle\hat{a}^{\dagger}(t) \hat{a}(t)\right\rangle-\lambda\left\langle\hat{b}^{\dagger}(t) \hat{b}(t)\right\rangle-$

$-\kappa\langle\hat{a}(t) \hat{b}(t)\rangle-(\lambda+\kappa M)$,

$\frac{d}{d t}\left\langle\hat{a}^{2}(t)\right\rangle=-2 \lambda\left\langle\hat{a}(t) \hat{b}^{\dagger}(t)\right\rangle-\kappa\left\langle\hat{a}^{2}(t)\right\rangle$,

$\frac{d}{d t}\left\langle\hat{b}^{2}(t)\right\rangle=-2 \lambda\left\langle\hat{a}^{\dagger}(t) \hat{b}(t)\right\rangle-\kappa\left\langle\hat{b}^{2}(t)\right\rangle$.

1. D.F. Walls. Squeezed states of light. Nature 306, 141 (1983).

2. G.J. Milburn, D.F. Walls. Production of squeezed states in a degenerate parametric amplifier. Opt. Commun. 39, 401 (1981).

3. Fesseha Kassahun. Refind Quantum Analysis of Light (Create Space Independent publishing Platform, 2014).

4. M.J. Collett, C.W. Gardiner. Squeezing of intracavity and traveling-wave light fields produced in parametric amplification. Phys. Rev. A 30, 1386 (1984).

5. B. Daniel, K. Fesseha. The propagator formulation of the degenerate parametric oscillator. Opt. Commun. 151, 384 (1998).

6. L.A. Lugiato, G. Strini. On the squeezing obtainable in parametric oscillators and bistable absorption. Opt. Comm. 41, 67 (1982).

7. S. Tesfa. Two-mode squeezing in a coherently driven degenerate parametric down conversion. Eur. Phys. J. D 46 , 351 (2008).

8. B. Teklu. Parametric oscillation with the cavity mode driven by coherent light and coupled to a squeezed vacuum reservoir. Opt. Commun. 261, 310 (2006).

ISSN 2071-0194. Ukr. J. Phys. 2021. Vol. 66, No. 8 
9. P.D. Drummond, K. Dechoum, S. Chaturvedi. Critical quantum fluctuations in the degenerate parametric oscillator. Phys. Rev. A 65, 033806 (2002).

10. S. Chaturvedi, K. Dechoum, P.D. Drummond. Limits to squeezing in the degenerate optical parametric oscillator. Phys. Rev. A 65, 033805 (2002).

11. Daniel Erenso. Enhanced squeezing in the transmitted fields in parametric oscillation with injected squeezed light at the pump frequency. Opt.Soc. Am. B 24, 867 (2007).

12. T. Abebe. The quantum analysis of nondegenerate threelevel laser with spontaneous emission and noiseless vacuum reservoir. Ukr. J. Phys. 63, 969 (2018).

13. M. Molla Gessesse. The noise effect of vacuum reservoir on the dynamics of three-level laser pumped by coherent light. Ukr. J. Phys. 65, 385 (2020).

14. C.M. Caves. Quantum-mechanical noise in an interferometer. Phys. Rev. D 23, 1693 (1981).

15. J.M. Xiao, L.A. WU, H.J. Kimble. Precision measurement beyond the shot-noise limit. Phys. Rev. Lett. 59, 278 (1987).

16. J. Gea-Banacloche, G. Leuchs. Applying squeezed states to nonideal interferometers. Opt. Soc. Am. B 4, 1667 (1987).

17. A.F. Pace, M.J. Collet, D.F. Walls. Quantum limits in interferometric detection of gravitational radiation. Phys. Rev. A 47, 3173 (1993).

18. A.S. Lane, M.D. Reid, D.F. Walls. Absorption spectroscopy beyond the shot-noise limit. Phys. Rev. Lett. 60, 1940 (1988).

19. L. Wu, M. Xia, H.J. Kimble. Squeezed states of light from an optical parametric oscillator. J. Opt. Soc. Am. B $\mathbf{4}$, 1465 (1987)

20. S. Lloyd, S.L. Braunstein. Quantum computation over continuous variables. Phys. Rev. Lett. 82, 1784 (1999).

21. S.L. Braunstein, H.J. Kimble. Dense coding for continuous variables. Phys. Rev. A 61, 042302 (2000).

22. J.M. Liu, B.S. Shi, X.F. Fan, J. Li, G.C. Guo. Wigner function description of continuous variable entanglement swapping. J. Opt. B: Quant. Semiclass. Opt. 3, 189 (2001).

23. T.C. Ralph. Continuous variable quantum cryptography. Phys. Rev. A 61, 010303 (1999).

24. Y. Zhang, H. Wang, X. Li, J. Jing, C. Xie, K. Peng. Experimental generation of bright two-mode quadrature squeezed light from a narrow-band nondegenerate optical parametric amplifier. Phys. Rev. A 62, 023813 (2000).

25. E. Alebachew. Continuous-variable entanglement in a nondegenerate three-level laser with a parametric oscillator. Phys. Rev. A 76, 023808 (2007).

26. S. Tesfa. Entanglement amplification in a nondegenerate three-level cascade laser. Phys. Rev. A 74, 043816 (2006).

27. M. Hillery, M.S. Zubairy. Entanglement conditions for twomode states. Phys. Rev. Lett. 96, 050503 (2006).

28. L.M. Duan, G. Giedke, J.I. Cirac, P. Zoller. Inseparability criterion for continuous variable systems. Phys. Rev. Lett. 84, 2722 (2000).

29. W.H. Louisell. Quantum Statistical Properties of Radiation (Wiley, 1973).

30. R. Simon. Peres-Horodecki separability criterion for continuous variable systems. Phys. Rev. Lett. 84, 2726 (2000).

31. G. Adesso, A. Serafini, F. Illuminati. Extremal entanglement and mixedness in continuous variable systems. Phys. Rev. A 70, 022318 (2004).

Received 07.09.20

\section{Д. Аєxy}

\section{ГЕНЕРАЦІЯ ДВОМОДОВОГО}

\section{СТИСНУТОГО І ПЕРЕПЛУТАНОГО СВІТЛА} ПРИ ПАРАМЕТРИЧНИХ КОЛИВАННЯХ

Грунтуючись на розв'язках квантових рівнянь Ланжевена, ми досліджували статистичне і квадратурне стиснення разом із властивістю змішування двомодового світла, що генерується невиродженими параметричними коливаннями, взаємодіючими 3 двомодовим стискаючим вакуумним резервуаром. Знайдено, що двомодове світло проявляе властивості двомодового стиснення і змішування в усьому часовому інтервалі. Показано, що наявність стискаючого вакуумного резервуара і зростання амплітуди накачування збільшують ступінь двомодового стиснення і змішування. Ми також показали, що стиснення і переплутування істотні в області, де середня кількість фотонів велика, а кореляція числа фотонів мала.

Клю чо в $i$ слов в: двомодове світло, двомодове стиснення, переплутування, кореляція числа фотонів. 